Methods: We used the Parent/Caregiver form in order to assess psychometric characteristics of the VABS-II and to develop norms for the French population. VABS-II comprises 4 domains, 11 subdomains, and an optional maladaptive behavior index. The French translation of the VABS-II followed standard cross-cultural translation methods. The study was performed in the Rhône-Alpes-Auvergne department comprising $12 \%$ of France inhabitants and well representing the French general population.

Results: From 4576 VABS-II questionnaires distributed, 1707 were returned and 1654 were analyzed. The reason for exclusion was the impossibility to score one of the subdomains. From 174 questionnaires included in the test-retest, 95 were analyzed, and 79 questionnaires were excluded because 86 under 34 days, $8>3$ months, and one participant that changed age group between test and retest. Scores based on French norms fluctuated around values based on US norms on all subdomains.

Conclusions: The French Vineland questionnaire is the single test with the adequate norms to allow identifying children with adaptive behavior difficulties. It should be used as a complement of the assessment of the intellectual quotient, according to DSM V, for the diagnosis of intellectual disabilities. It must be done in reference to the developmental and cultural standards specific to the environment in which the person is evolving.

Disclosure: No significant relationships.

Keywords: autism spectrum disorder; French norms; adaptative behavior; validation

\section{Schizophrenia and other psychotic disorders}

\section{8}

\section{Drug attitude inventory is relevant to LAI treatment persistence in schizophrenia: Preliminary results}

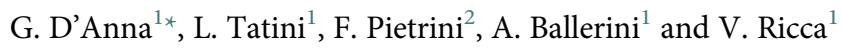

${ }^{1}$ Department Of Health Sciences, University of Florence, Florence, Italy and ${ }^{2}$ Department Of Mental Health And Addictions, Central Tuscany NHS Trust, Florence, Italy

${ }^{*}$ Corresponding author.

doi: 10.1192/j.eurpsy.2021.422

Introduction: Patients' attitudes and subjective experience are crucial in the management of severe mental illness, but their practical value is overlooked.

Objectives: To identify predictors of future adherence to LAI antipsychotic maintenance treatment of schizophrenia among socio-demographic, clinical, and psychometric characteristics including Drug Attitude Inventory-10 (DAI-10) and Subjective Well-being under Neuroleptics short form (SWN-K) scores.

Methods: Retrospective baseline data from 53 clinically stable outpatients with schizophrenia switched from oral to LAI therapy were collected. Patients continuing treatment at the time of analysis $(n=29)$ were compared to those who had discontinued it $(n=24)$. Selected variables were further evaluated in survival analyses.

\begin{tabular}{llll}
\hline & $\begin{array}{c}\text { Continued } \\
\text { treatment }\end{array}$ & $\begin{array}{c}\text { Discontinued } \\
\text { treatment }\end{array}$ & $\chi^{2}$ or t \\
\hline $\begin{array}{l}\text { Treatment persistence } \\
\text { (months) }\end{array}$ & $63.79 \pm 21.01$ & $23.88 \pm 25.80$ & $6.21^{\star \star}$ \\
\hline Age (years) & $39.17 \pm 10.11$ & $35.58 \pm 13.39$ & 1.11 \\
\hline Male & $15(51.7 \%)$ & $13(54.1 \%)$ & 0.03 \\
\hline Single & $20(69.0 \%)$ & $15(62.5 \%)$ & 0.25 \\
\hline Instruction (years) & $13.28 \pm 3.31$ & $11.83 \pm 3.56$ & 1.53 \\
\hline Employed & $20(69.0 \%)$ & $7(29.2 \%)$ & $8.32^{\star \star}$ \\
\hline Illness duration (years) & $17.69 \pm 10.53$ & $13.42 \pm 11.36$ & 1.42 \\
\hline Previous hospitalisations & $2.10 \pm 1.32$ & $2.67 \pm 1.86$ & -1.29 \\
\hline MADRS & $13.59 \pm 9.06$ & $14.67 \pm 8.99$ & -0.43 \\
\hline YMRS & $5.52 \pm 5.57$ & $6.00 \pm 9.94$ & -0.22 \\
\hline p-PANSS & $12.17 \pm 5.20$ & $14.38 \pm 6.13$ & -1.41 \\
\hline n-PANSS & $10.90 \pm 5.39$ & $15.63 \pm 7.93$ & $-2.48^{\star}$ \\
\hline g-PANSS & $29.38 \pm 10.33$ & $33.63 \pm 10.26$ & -1.49 \\
\hline PANSS & $52.66 \pm 17.57$ & $63.96 \pm 20.61$ & $-2.15^{\star}$ \\
\hline DAI-10 & $3.86 \pm 4.96$ & $-1.13 \pm 5.80$ & $3.38^{\star \star}$ \\
\hline SWN-K & $74.93 \pm 23.07$ & $81.00 \pm 15.60$ & -1.09 \\
\hline & & & \\
\hline
\end{tabular}

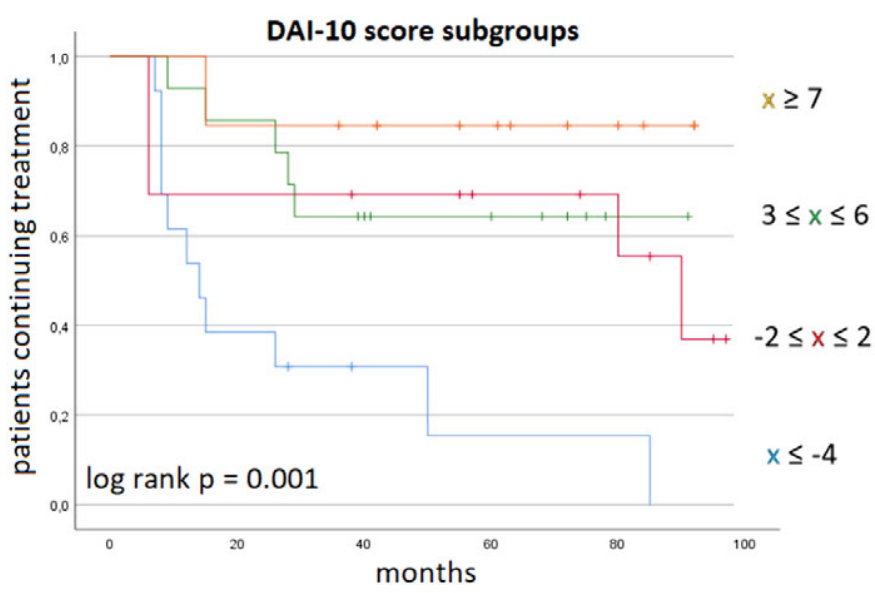

Results: Between-group differences are presented in Table $1{ }^{* *}$ : $\left.\mathrm{p}<0.01{ }^{*}: \mathrm{p}<0.05\right)$.

Cox regression analysis included instruction, employment, hospitalisations, PANSS subscales and DAI-10 scores: a protective role against treatment discontinuation was outlined only for employment (HR 0.16; 95\%CI 0.05-0.50) and higher DAI-10 scores (HR 0.85; 95\%CI 0.78-0.94). DAI-10 scores delineated distinct adherence trajectories (Figure 1).

Conclusions: Baseline DAI-10 scores may identify patients at risk of dropout after switching to LAI.

Disclosure: No significant relationships.

Keywords: Drug Attitude Inventory; adherence; schizophrénia; LAI 